\title{
Hydromechanics of low-Reynolds-number flow. Part 4. Translation of spheroids
}

\author{
By ALLEN T. CHWANG AND THEODORE Y. WU \\ Engineering Science Dopartment, California Institute of Technology, Pasadena
}

(Received 29 Septembor 1975)

The problem of a uniform transverse flow past a prolate spheroid of arbitrary aspect ratio at low Reynolds numbers has been analysed by the method of matched asymptotic expansions. The solution is found to depend on two Reynolds numbers, one based on the semi-minor axis $b, R_{b}=U b / v$, and the other on the semi-major axis $a, R_{a}=U a / \nu$ ( $U$ being the free-stream velocity at infinity, which is perpendicular to the major axis of the spheroid, and $v$ the kinematic viscosity of the fluid). A drag formula is obtained for small values of $R_{b}$ and arbitrary values of $R_{a}$. When $R_{a}$ is also small, the present drag formula reduces to the Oberbeck (1876) result for Stokes flow past a spheroid, and it gives the Oseen (1910) drag for an infinitely long cylinder when $R_{a}$ tends to infinity. This result thus provides a clear physical picture and explanation of the 'Stokes paradox' known in viscous flow theory.

\section{Introduction}

Viscous flow past a sphere and a circular cylinder at very low Reynolds numbers has been analysed by Stokes (1851) and Oseen (1910) in the framework of the Stokes and Oseen equations respectively. There is a fundamental difference between these two solutions. In the case of a sphere, the problem is threo-dimensional, and the well-known Stokes drag formula $D=6 \pi \mu U a$ ( $U$ is the free-stream velocity at infinity, $a$ the radius of the sphere and $\mu$ the dynamic viscosity coefficient of the fluid) does not depend on the Reynolds number $R_{a}=U a / \nu$, where the kinematic viscosity coefficient $\nu$ is related to the density of the fluid $\rho$ and $\mu$ by $\nu=\mu / \rho$. In other words, the Stokes solution is valid at zero Reynolds number. However, in the case of a circular cylinder, it is impossible to construct a solution using Stokes' equations. The non-existence of a Stokes solution for unbounded flow past any two-dimensional body is often referred to as Stokes' paradox. In order to find a solution for uniform flow of velocity $U$ past a circular cylinder of radius $b$, one has to apply Oseen's equations, which incorporate a linearized inertial term. Hence the famous Oseen drag formula for a circular cylinder (force per unit length),

$$
D_{\mathrm{2D}}=\frac{4 \pi \mu U}{\log \left(4 / R_{b}\right)-\gamma+\frac{1}{2}}
$$

$\left(\gamma=0.5772 \ldots\right.$ is Euler's constant), depends on the Reynolds number $R_{b}=U b / \nu$. Proudman \& Pearson (1957) and Kaplun \& Lagerstrom (1957) have related Stokes' and Oseen's solutions to the asymptotic expansions of the Navier-Stokes equations. They demonstrated that it is possible to obtain higher-order approximations to the flow past a sphere and a circular cylinder by applying the-so-called 
'matched asymptotic expansion' technique. In order to clarify the differences between Stokes' and Oseen's drag formulae and to study in detail the transition from the three-dimensional case to the two-dimensional case, it is of great value to investigate the problem of uniform transverse flow past a prolate ellipsoid of revolution, or a prolate spheroid with semi-major axis $a$ and semi-minor axis $b$. Oberbeck (1876) first obtained the solution for an ellipsoid at any orientation to a uniform flow using the Stokes equations. Breach (1961), following Proudman \& Pearson's technique, has extended Oberbeck's result to include some inertial effect for uniform flow past an ellipsoid of revolution with its axis of revolution parallel to the free stream at infinity such that a Stokes stream function $\psi$ may be used to construct the solution. His solution, however, is valid only when both $R_{a}$ and $R_{b}$ tend to zero. Shi (1965) analysed the problem of uniform flow past a prolate spheroid of large aspect ratio $(b / a \ll 1)$ with its axis of symmetry perpendicular to the undisturbed free stream at infinity. The assumption that the spheroid is of large aspect ratio, i.e. very slender, made it possible for Shi (1965) to break the inner expansions into a 'shank expansion' and two 'end expansions', which, in turn, were matched with the outer expansions. The leading term of his solution for the drag per unit length on a slender spheroid is the same as that of Oseen's for a circular cylinder. However, his solution is valid only when the Reynolds number $R_{a}$ based on the semi-major axis tends to infinity, as shown in $\S 3$ of the present paper.

We consider here the problom of transverse viscous flow past a prolate ellipsoid of revolution of arbitrary aspect ratio $(0<b / a \leqslant 1)$ with its axis of revolution perpendicular to the free-stream velocity at infinity. The Reynolds number $R_{b}$ based on the semi-minor axis is assumed to be small, but $R_{a}$ is arbitrary. In the inner expansion of the Navier-Stokes equations, we have applied the singularity method to construct an exact solution of Stokes' equations for viscous flow past a prolate spheroid in terms of a line distribution of Stokeslets and potential doublets (see $\$ 3$ ). The method of singularities proves to be very effective in determining the inner expansions. For more information regarding the construction of exact solutions in Stokes flows refer to Chwang \& Wu $(1974,1975)$ and Chwang (1975). The leading term of the outer expansions is constructed using a line distribution of constant Oseenlets. Thus we neglect the end effect in the leading term. However, by matching the inner limit of the outer expansions with the outer limit of the inner expansions at the mid-plane (see $\$ 3$, case 2 ), where the end effect reduces to a minimum or even disappears altogether because of the symmetry of the body and of the flow, we obtain a drag formula in closed analytic form which seems quite satisfactory in comparison with the Oberbock (1876) drag formula when both $R_{b}$ and $R_{a}$ tend to zero and with the Oseen (1910) result for a circular oylinder when $R_{b}$ tends to zero and $R_{a}$ tends to infinity.

\section{The Stokes and Oseen expansions}

We shall consider in this paper the problem of viscous flow with uniform freestream velocity $U$ in the $y$ direction past an ellipsoid of revolution

$$
x^{2} / a^{2}+r^{2} / b^{2}=1 \quad\left(r^{2}=y^{2}+z^{2}, a \geqslant b\right),
$$


, where the focal length $2 c$ and eccentricity $e$ are related by

$$
c=\left(a^{2}-b^{2}\right)^{\frac{1}{2}}=e a \quad(0 \leqslant e<1) .
$$

The governing differential equations for a viscous incompressible fluid are the Navier-Stokes equations

$$
\begin{gathered}
\nabla \cdot \mathbf{u}=\mathbf{0}, \\
(\mathbf{u} . \nabla) \mathbf{u}=-\rho^{-1} \nabla p+\nu \nabla^{2} \mathbf{u},
\end{gathered}
$$

where $p$ is the pressure, $\mathbf{u}=u \mathbf{e}_{x}+v \mathbf{e}_{y}+w \mathbf{e}_{z}$ the velocity vector, $\rho$ the density and $v$ the constant kinematic viscosity coefficient of the fluid, $\mathbf{e}_{x}, \mathbf{e}_{y}$ and $\mathbf{e}_{z}$ being unit vectors in the $x, y$ and $z$ directions respectively. Introducing the inner or Stokes variables

$$
x^{*}=x / a, \quad y^{*}=y / b, \quad z^{*}=z / b,
$$

and non-dimensionalizing the velocity vector and pressure field by setting

$$
\mathbf{u}^{*}=\mathbf{u} / U, \quad p^{*}=b\left(p-p_{\infty}\right) / \mu U,
$$

we obtain from (2) the dimensionless Navier-Stokes equations in inner variables as

$$
\frac{b}{a} \frac{\partial u^{*}}{\partial x^{*}}+\frac{\partial v^{*}}{\partial y^{*}}+\frac{\partial w^{*}}{\partial z^{*}}=0
$$

$$
\begin{array}{r}
R_{b}\left(\frac{b}{a} u^{*} \frac{\partial}{\partial x^{*}}+v^{*} \frac{\partial}{\partial y^{*}}+w^{*} \frac{\partial}{\partial z^{*}}\right) \mathbf{u}^{*}=-\left(\frac{b}{a} \mathbf{e}_{x} \frac{\partial}{\partial x^{*}}+\mathbf{e}_{y} \frac{\partial}{\partial y^{*}}+\mathbf{e}_{z} \frac{\partial}{\partial z^{*}}\right) p^{*} \\
+\left(\frac{b^{2}}{a^{2}} \frac{\partial^{2}}{\partial x^{* 2}}+\frac{\partial^{2}}{\partial y^{* 2}}+\frac{\partial^{2}}{\partial z^{* 2}}\right) \mathbf{u}^{*}
\end{array}
$$

where $p_{\infty}$ is the constant pressure at infinity, $\mu(=\rho \nu)$ the dynamic viscosity coefficient and $R_{b}$ the Reynolds number based on the semi-minor axis $b$, i.e.

$$
R_{b}=U b / v \text {. }
$$

If we introduce another Reynolds number based on the semi-major axis $a$,

$$
R_{a}=U a / \nu
$$

the axis ratio $b / a$ in (5) can also be expressed as the ratio of the two Reynolds numbers: $b / a=R_{b} / R_{a}$.

When the Reynolds number $R_{b}$ based on $b$ tends to zero and the Reynolds number based on $a$ is arbitrary, i.e.

$$
\infty>R_{a} \geqslant R_{b} \rightarrow 0,
$$

we may assume the inner expansions for velocity and pressure to have the form (following the ideas of Kaplun \& Lagerstrom 1957)

$$
\begin{aligned}
& \mathbf{u}^{*}=\mathbf{u}_{0}^{*}+\mathbf{u}_{1}^{*}+\mathbf{u}_{2}^{*}+\ldots, \\
& p^{*}=p_{0}^{*}+p_{1}^{*}+p_{2}^{*}+\ldots,
\end{aligned}
$$

where $\mathbf{u}_{0}^{*}, p_{0}^{*}$, etc. are functions of $x^{*}, y^{*}, z^{*}, R_{a}$ and $R_{b}$, and

$$
\mathbf{u}_{n+1}^{*} / \mathbf{u}_{n}^{*} \rightarrow 0, \quad p_{n+1}^{*} / p_{n}^{*} \rightarrow 0 \quad \text { as } \quad R_{b} \rightarrow 0 .
$$


The expansions (9) are required to satisfy the differential equations (5) and the no-slip boundary condition on the surface of the spheroid, given by (1), i.e.

$$
\mathbf{u}^{*}=0 \quad \text { on } \quad x^{* 2}+r^{* 2}=1 \quad\left(r^{* 2}=y^{* 2}+z^{* 2}\right) .
$$

Since the inner expansions are invalid at large values of $R^{*} \equiv\left(x^{* 2}+r^{* 2}\right)^{\frac{1}{2}}$, the uniform free-stream condition at infinity does not have to be satisfied. Instead, it should be replaced by the requirement that the inner (or Stokes) expansions must match outer (or Oseen) expansions which are valid at infinity. Obviously, the leading terms of the inner expansions (9) will satisfy (5) with the left-hand side of $(5 b)$ replaced by zero, or the Stokes equations

$$
\begin{gathered}
\nabla . \mathbf{u}_{0}=0, \\
\nabla p_{0}=\mu \nabla^{2} \mathbf{u}_{0},
\end{gathered}
$$

if we return to dimensional variables.

Introducing the outer or Oseen variables

$$
\tilde{x}=U x / \nu=R_{a} x^{*}, \quad \tilde{y}=R_{b} y^{*}, \quad \tilde{z}=R_{b} z^{*},
$$

and scaling the pressure by

$$
\tilde{p}=\left(p-p_{\infty}\right) / \rho U^{2},
$$

we obtain from (2) the dimensionless Navier-Stokes equations in outer variables as

$$
\begin{gathered}
\tilde{\nabla} \cdot \mathbf{u}^{*}=\mathbf{0}, \\
\left(\mathbf{u}^{*} \cdot \tilde{\nabla}\right) \mathbf{u}^{*}=-\tilde{\nabla} \tilde{p}+\tilde{\nabla}^{2} \mathbf{u}^{*},
\end{gathered}
$$

where $\tilde{\nabla}$ and $\tilde{\nabla}^{2}$ are in terms of outer variables and $\mathbf{u}^{*}$ is scaled by (4). The surface of the prolate spheroid (1) becomes

$$
(b \tilde{x} / a)^{2}+\tilde{r}^{2}=R_{b}^{2} \quad\left(\tilde{r}^{2}=\tilde{y}^{2}+\tilde{z}^{2}\right) .
$$

As the Reynolds number $R_{b}$ tends to zero, the body shrinks to a needle of zero radius. When $b=a$, this needle further reduces to a point. For arbitrary values of $b / a(0<b / a \leqslant 1)$, it is a needle of finite length. A needle of zero radius, in this outer limit, cannot cause a finite disturbance in the fluid. Hence the velocity vector $\mathbf{u}^{*}$ at any fixed point will tend to the free-stream value $\mathbf{e}_{y}$. Thus we assume the Oseen expansions for velocity and pressure to be of the form

$$
\begin{gathered}
\mathbf{u}^{*}=\mathbf{e}_{y}+\mathbf{q}_{1}+\mathbf{q}_{2}+\ldots, \\
\tilde{p}=\tilde{p}_{1}+\tilde{p}_{2}+\ldots,
\end{gathered}
$$

where

$$
\mathbf{q}_{n+1} / \mathbf{q}_{n} \rightarrow 0, \quad \tilde{p}_{n+1} / \tilde{p}_{n} \rightarrow 0 \text { as } R_{b} \rightarrow 0 .
$$

Substituting (15) into (13) we see that the leading terms of the Oseen expansions $\mathbf{q}_{1}$ and $\tilde{p}_{1}$ satisfy the Oseen equations, namely

$$
\begin{gathered}
\tilde{\nabla} \cdot \mathbf{q}_{1}=0, \\
\partial \mathbf{q}_{1} / \partial \tilde{y}=-\tilde{\nabla} \tilde{p}_{1}+\tilde{\nabla}^{2} \mathbf{q}_{1} .
\end{gathered}
$$

The outer expansions must satisfy the free-stream condition at infinity, hence all the $\mathbf{q}_{i}$ 's $(i=1,2,3, \ldots)$ must vanish at infinity. However, the inner limits of 
these outer expansions need not satisfy the no-slip boundary condition. They are only required to match the outer limits of the inner expansions.

\section{Determination of solutions}

Before we discuss the general solution for arbitrary values of $R_{a}$, we first study the limiting case where $R_{a}$, as well as $R_{b}$, is very small.

\section{Case 1. $R_{a}$ small}

In this limiting case, both the Reynolds numbers $R_{b}$ and $R_{a}$ are very small:

$$
0<R_{b} \leqslant R_{a} \ll 1, \quad R_{b}=O\left(R_{a}\right) .
$$

The leading terms of the inner expansions can be obtained from the known Stokes solutions for uniform transverse flow past a prolate spheroid (see Oberbock 1876; Chwang \& Wu 1975). Using the singularity method, the solution of (11) satisfying the no-slip boundary condition (10) may be constructed from a line distribution of Stokeslets with constant density and potential doublets with parabolic density between the foci $x=-c$ and $c$ (see equations (22)-(27) of Chwang \& Wu 1975):

where

$$
\mathbf{u}_{0}^{*}=\left(\alpha / \alpha_{0}\right) \mathbf{h}_{0}, \quad p_{0}^{*}=\left(\alpha / \alpha_{0}\right) s_{0},
$$

$$
\begin{aligned}
\mathbf{h}_{0} & =\mathbf{e}_{y}-\alpha_{0} \int_{-c}^{c}\left(\frac{\mathbf{e}_{y}}{R_{\xi}}+\frac{y \mathbf{R}_{\xi}}{R_{\xi}^{3}}\right) d \xi+\beta_{0} \int_{-c}^{c}\left(1-\frac{\xi^{2}}{c^{2}}\right) \nabla\left[\frac{\partial}{\partial y}\left(\frac{1}{R_{\xi}}\right)\right] d \xi, \\
s_{0} & =2 b \alpha_{0} \int_{-c}^{c} \frac{\partial}{\partial y}\left(\frac{1}{R_{\xi}}\right) d \xi \\
R_{\xi} & =\left|\mathbf{R}_{\xi}\right|, \quad \mathbf{R}_{\xi}=(x-\xi) \mathbf{e}_{x}+y \mathbf{e}_{y}+z \mathbf{e}_{z}, \\
\alpha_{0} & =2 \beta_{0} b^{-2}=2 e^{2}\left[2 e+\left(3 e^{2}-1\right) \log \frac{1+e}{1-e}\right]^{-1} .
\end{aligned}
$$

The arbitrary constant $\alpha$, which may depend on $R_{a}$ and $R_{b}$, but not on $x^{*}, y^{*}$ and $z^{*}$, has yet to be determined from the condition at infinity. As $R^{*}\left(\equiv\left(x^{* 2}+r^{* 2}\right)^{\frac{1}{2}}\right)$ tends to infinity, the velocity $\mathbf{u}_{0}^{*}$ tends, in terms of the outer variables, to

$$
\lim _{R^{*} \rightarrow \infty} \mathbf{u}_{0}^{*}=\frac{\alpha}{\alpha_{0}}\left[\mathbf{e}_{y}-\alpha_{0} \int_{-e \mathbf{R}_{a}}^{e R_{a}}\left(\frac{\mathbf{e}_{y}}{\tilde{\hat{R}}_{\xi}}+\frac{\tilde{y} \tilde{\mathbf{R}}_{\xi}}{\tilde{R}_{\xi}^{3}}\right) d \tilde{\xi}+O\left(\frac{1}{R^{* 3}}\right)\right],
$$

where

$$
\tilde{\xi}=U \xi / \nu, \quad \tilde{R}_{\xi}=\left|\tilde{\mathbf{R}}_{\xi}\right|, \quad \tilde{\mathbf{R}}_{\xi}=U \mathbf{R}_{\xi} / \nu .
$$

When the Reynolds number $R_{a}$ is also very small, $(19 a)$ further reduces to

$$
\lim _{R^{*} \rightarrow \infty, R_{a} \rightarrow 0} \mathbf{u}_{0}^{*}=\frac{\alpha}{\alpha_{0}}\left[\mathbf{e}_{y}-2 e \alpha_{0} R_{a}\left(\frac{\mathbf{e}_{y}}{\tilde{R}}+\frac{\tilde{y} \tilde{\mathbf{R}}}{\tilde{R}^{3}}\right)+O\left(R_{a}^{2}, R_{a} / R^{* 2}\right\rangle\right],
$$

where

$$
\tilde{\boldsymbol{R}}=|\tilde{\mathbf{R}}|, \quad \tilde{\mathbf{R}}=\tilde{x} \mathbf{e}_{x}+\tilde{y} \mathbf{e}_{y}+\tilde{z} \mathbf{e}_{z} .
$$

Comparing (15a) with $(20 a)$, we conclude that

$$
\alpha=\alpha_{0} .
$$


The second term on the right-hand side of $(20 a)$ has to match the outer expansions $\mathbf{u}^{*}-\mathrm{e}_{y}$ as $R_{b} \rightarrow 0$ and $R_{a} \rightarrow 0$. A solution of (16) which meets the above requirement and which vanishes at infinity is a line distribution of constant Oseenlets. Thus

$$
\begin{gathered}
\mathbf{q}_{1}=-2 \alpha \mathbf{e}_{y} \int_{-e R_{a}}^{e R_{a}} \frac{\exp \left[-\frac{1}{2}\left(\tilde{R}_{\xi}-\tilde{y}\right)\right]}{\tilde{R}_{\xi}} d \tilde{\xi}+2 \alpha \tilde{\nabla} \int_{-e R_{a}}^{e R_{a}}\left\{\frac{\exp \left[-\frac{1}{2}\left(\tilde{R}_{\xi}-\tilde{y}\right)\right]}{\tilde{R}_{\xi}}-\frac{1}{\widetilde{R}_{\xi}}\right\} d \tilde{\xi}, \\
\tilde{p}_{1}=2 \alpha \int_{-e R_{a}}^{e R_{a}} \frac{\partial}{\partial \tilde{y}}\left(\frac{1}{\tilde{R}_{\xi}}\right) d \tilde{\xi} .
\end{gathered}
$$

We note that $\tilde{p}_{1}$ in dimensional form is exactly the same as $p_{0}^{*}$ as given by the inner expansions (18). As $\widetilde{R} \rightarrow 0$ and $R_{a} \rightarrow 0$, the velocity $\mathbf{q}_{1}$ reduces to

$\lim _{\tilde{R} \rightarrow 0 . R_{a} \rightarrow 0} \mathbf{q}_{1}=2 e \alpha R_{a}\left[-\left(\frac{\mathbf{e}_{y}}{\tilde{R}}+\frac{\tilde{y} \tilde{\mathbf{R}}}{\tilde{R}^{3}}\right)+\frac{1}{2} \mathbf{e}_{y}-\frac{1}{4} \frac{\partial}{\partial \tilde{y}}\left(3 \tilde{R} \mathbf{e}_{y}-\frac{\tilde{y} \tilde{\mathbf{R}}}{\tilde{R}}\right)\right]$

$$
+O\left(R_{a}^{2}, R_{a} \tilde{R}\right) \text {. }
$$

Thus the first term on the right-hand side of (23) matches the second term on the right-hand side of (20a) perfectly. The remaining terms in (23) have to be matched with the outer limit of the inner expansions $\mathbf{u}^{*}-\mathbf{u}_{0}^{*}$ as $R_{b} \rightarrow 0$ and $R_{a} \rightarrow 0$.

The solution $\mathbf{u}_{1}^{*}\left(\equiv u_{1}^{*} \mathrm{e}_{x}+v_{1}^{*} \mathbf{e}_{y}+w_{1}^{*} \mathbf{e}_{z}\right)$ which satisfies the equations

$$
\begin{aligned}
\frac{b}{a} \frac{\partial u_{1}^{*}}{\partial x^{*}}+ & \frac{\partial v_{1}^{*}}{\partial y^{*}}+\frac{\partial w_{1}^{*}}{\partial z^{*}}=0 \\
-\left(\frac{b}{a} \mathbf{e}_{x} \frac{\partial}{\partial x^{*}}+\mathbf{e}_{y} \frac{\partial}{\partial y^{*}}+\mathbf{e}_{z} \frac{\partial}{\partial z^{*}}\right) p_{1}^{*} & +\left(\frac{b^{2}}{a^{2}} \frac{\partial^{2}}{\partial x^{* 2}}+\frac{\partial^{2}}{\partial y^{* 2}}+\frac{\partial^{2}}{\partial z^{* 2}}\right) \mathbf{u}_{1}^{*} \\
& =R_{a} \frac{b}{a}\left(\frac{b}{a} u_{0}^{*} \frac{\partial}{\partial x^{*}}+v_{0}^{*} \frac{\partial}{\partial y^{*}}+w_{0}^{*} \frac{\partial}{\partial z^{*}}\right) \mathbf{u}_{0}^{*}
\end{aligned}
$$

and the no-slip boundary condition (10) can be constructed with the help of (23) as

$$
\mathbf{u}_{1}^{*}=e \alpha R_{a} \mathbf{h}_{0}+\mathbf{u}_{1, \mathrm{PI}}^{*},
$$

where the particular integral $u_{1, \text { PI }}^{*}$ satisfies $(24 b)$ and has the following limiting property:

$$
\lim _{R^{*} \rightarrow \infty, R_{a} \rightarrow 0} \mathbf{u}_{1, \mathrm{PI}}^{*} \sim-\frac{1}{2} e \alpha R_{a} \frac{\partial}{\partial \tilde{y}}\left(3 \tilde{R} \mathbf{e}_{y}-\tilde{y} \tilde{\mathbf{R}} / \tilde{R}\right) .
$$

Because of the antisymmetry of $\mathbf{u}_{1, \mathrm{PI}}^{*}$ and the symmetry of the spheroid (1) with respect to the plane $y=0$, it contributes nothing to the total drag force acting on the ellipsoid of rovolution (1).

By (18), (21) and (25), the total drag force (in the $y$ direction) can be evaluated from the total strength of Stokeslets as

or

$$
D=8 \pi \mu U \int_{-c}^{c} \alpha_{0}\left[1+e \alpha_{0} R_{a}+O\left(R_{a}^{2}\right)\right] d \xi,
$$

$$
\begin{array}{r}
D=\frac{32 \pi \mu U a e^{3}}{2 e+\left(3 e^{2}-1\right) \log [(1+e) /(1-e)]}\left[1+\frac{2 e^{3} R_{a}}{2 e+\left(3 e^{2}-1\right) \log [(1+e) /(1-e)]}\right. \\
\left.+O\left(R_{a}^{2}\right)\right] .
\end{array}
$$


We note that the leading term in $(26 b)$ is precisely the Oberbeck drag formula derived from the Stokes equations (see also Chwang \& Wu 1975). If we let $b / a \rightarrow 1$ or equivalently let $e \rightarrow 0$ we have from $(26 b)$ that

$$
\lim _{b / a \rightarrow 1} D=6 \pi \mu U a\left[1+\frac{3}{8} R_{a}+O\left(R_{a}^{2}\right)\right],
$$

which is exactly the Oseen (1910) correction to the Stokes drag formula for a sphere.

\section{Case 2. $R_{a}$ arbitrary}

In this general case we shall only assume $R_{b}$ small, leaving $R_{a}$ arbitrary:

$$
0<R_{b} \ll 1, \quad R_{a} \text { arbitrary. }
$$

The leading term $\mathbf{u}_{0}^{*}$ which satisfies the Stokes equations (11) and the no-slip boundary condition (10) is again given by (18) with the arbitrary constant $\alpha$ to be determined by matching with the outer expansions. We note that $\alpha$ may still depend on $R_{a}$ and $R_{b}$, but definitely not on $x^{*}, y^{*}$ and $z^{*}$. As $R^{*}$ approaches infinity, the outer limit of $\mathbf{u}_{0}^{*}$ is given, in terms of the outer variables, by

$$
\begin{aligned}
\lim _{R^{*} \rightarrow \infty} \mathbf{u}_{0}^{*}= & \frac{\alpha}{\alpha_{0}} \mathbf{e}_{y}\left[1+\frac{\alpha_{0} \tilde{y}^{2}}{\tilde{r}^{2}}\left(\frac{\tilde{x}-e R_{a}}{\widetilde{Q}_{2}}-\frac{\tilde{x}+e R_{a}}{\widetilde{Q}_{1}}\right)-\alpha_{0} \log \frac{\widetilde{Q}_{2}-\left(\tilde{x}-e R_{a}\right)}{\tilde{Q}_{1}-\left(\tilde{x}+e R_{a}\right)}\right] \\
& +\alpha \tilde{y} \mathbf{e}_{x}\left(\frac{1}{\tilde{Q}_{1}}-\frac{1}{\tilde{Q}_{2}}\right)+\frac{\alpha \tilde{y} \tilde{z}}{\tilde{r}^{2}} \mathbf{e}_{z}\left(\frac{\tilde{x}-e R_{a}}{\tilde{Q}_{2}}-\frac{\tilde{x}+e R_{a}}{\tilde{Q}_{1}}\right)+O\left(\frac{1}{R^{* 3}}\right),
\end{aligned}
$$

where

$$
\tilde{Q}_{1}=U\left[(x+c)^{2}+r^{2}\right]^{\frac{1}{2}} / v, \quad \widetilde{Q}_{2}=U\left[(x-c)^{2}+r^{2}\right]^{\frac{1}{2}} / \nu .
$$

We note that $\left(\alpha / \alpha_{0}\right) \mathbf{e}_{y}$ is no longer the dominant term in (29) if $R_{a}$ is not small. Only when $R_{a}$ is very small does the right-hand side of $(29 a)$ tend to $\left(\alpha / \alpha_{0}\right) \mathrm{e}_{y}$.

Since the fundamental solution of (16) is an Oseenlet, the leading term of the outer expansion may be constructed from a line distribution of Oseenlets, whose strengths must be symmetric with respect to the plane $x=0$ because of the symmetry of the spheroid (1). When viewed in the outer variables, the body shrinks to a needle of zero radius and finite length when $R_{a}$ is arbitrary. When the needle is very short, i.e. when $R_{a}$ is very small, we see from case 1 that the Oseenlet distribution is of constant density. For a needle of finite length, we anticipate that the strengths of the Oseenlets will be fairly constant along the bulk of the needle; near the two ends the strengths have to be modified to account for the finiteness of the needle. From Shi's (1965) analysis we see that the end effect does not affect the leading term of the outer expansion; it shows up in the higher-order terms only. As a first approximation, we assume here that $\mathbf{q}_{1}$ is still given by $(22 a)$.

For $\tilde{r} \rightarrow 0$ and $-e R_{a}<\tilde{x}<e R_{a}$, the inner limit of $\mathbf{q}_{1}$ is obtained from (22a) as

$$
\begin{aligned}
\lim _{\tilde{r} \rightarrow 0} \mathbf{q}_{1}= & 2 \alpha \mathbf{e}_{y}\left[\gamma+\log \left(\frac{1}{4} \tilde{r}\right)+\frac{1}{2} E_{1}\left(\frac{1}{2} e R_{a}-\frac{1}{2} \tilde{x}\right)+\frac{1}{2} E_{1}\left(\frac{1}{2} e R_{a}+\frac{1}{2} \tilde{x}\right)\right] \\
& -2 \alpha \tilde{y} \tilde{\nabla}(\log \tilde{r})+2 \alpha \mathbf{e}_{x}\left\{\frac{\exp \left[-\frac{1}{2}\left(e R_{a}-\tilde{x}\right)\right]}{e R_{a}-\tilde{x}}-\frac{\exp \left[-\frac{1}{2}\left(e R_{a}+\tilde{x}\right)\right]}{e R_{a}+\tilde{x}}\right. \\
& \left.-\frac{1}{e R_{a}-\tilde{x}}+\frac{1}{e R_{a}+\tilde{x}}\right\}+O(\tilde{r} \log \tilde{r}),
\end{aligned}
$$


where $\gamma=0.5772 \ldots$ is Euler's constant and $E_{1}(\zeta)$ is the exponential integral defined by

$$
E_{1}(\zeta)=\int_{\zeta}^{\infty} \frac{e^{-t}}{t} d t
$$

For small values of $\zeta, E_{1}(\zeta)$ can be written as

$$
E_{1}(\zeta)=-\gamma-\log \zeta+e^{-\zeta} \sum_{m=1}^{\infty}\left(1+\frac{1}{2}+\ldots+\frac{1}{m}\right) \frac{\zeta^{m}}{m !},
$$

and for large values of $\zeta$, the asymptotic expansion of $E_{1}(\zeta)$ is

$$
E_{1}(\zeta)=\frac{e^{-\zeta}}{\zeta}\left(1-\frac{1 !}{\zeta}+\frac{2 !}{\zeta^{2}}+\ldots\right)
$$

Because of our neglect of the end effect in determining the outer expansion $\mathbf{q}_{1}$, we find that the inner limit of $q_{1}$ given by (30) cannot be matched perfectly with the outer limit of $\mathrm{u}_{0}^{*}$ given by (29) for arbitrary $\tilde{x}$ between $-e R_{a}$ and $e R_{a}$. However, we expect that our solution will be better if we apply it further away from the ends of the needle. The furthest such point, where the end effect reduces to its minimum or even disappears because of the symmetry of the body, is the centre of the needle, i.e. $\tilde{x}=0$. At $\tilde{x}=0,(29 a)$ becomes

$$
\lim _{R^{*} \rightarrow \infty} \mathbf{u}_{0}^{*}=\frac{\alpha}{\alpha_{0}} \mathbf{e}_{y}\left[1-\frac{2 \alpha_{0} \tilde{y}^{2}}{\tilde{r}^{2}}-2 \alpha_{0} \log \left(2 e R_{a} / \tilde{r}\right)\right]-\frac{2 \alpha \tilde{y} \tilde{z}}{\tilde{r}^{2}} \mathbf{e}_{z}+O\left(1 / R^{* 3}, \tilde{r}^{2}\right) \quad(\tilde{x}=0)
$$

and $(30)$ reduces to

$$
\lim _{\tilde{r} \rightarrow 0} \mathbf{q}_{1}=2 \alpha \mathbf{e}_{y}\left[\gamma+\log \left(\frac{1}{4} \tilde{r}\right)+E_{1}\left(\frac{1}{2} e R_{a}\right)\right]-2 \alpha \tilde{y} \tilde{\nabla}(\log \tilde{r})+O(\tilde{r} \log \tilde{r}) \quad(\tilde{x}=0) .
$$

From (32) and (33), we see that $\lim _{R^{*} \rightarrow \infty} \mathbf{u}_{0}^{*}$ will match $\mathbf{e}_{y}+\underset{\tilde{r} \rightarrow 0}{\lim _{1}} \mathbf{q}_{1}$ perfectly if

$$
\alpha^{-1}=\alpha_{0}^{-1}-2\left[\gamma+\log \left(\frac{1}{2} e R_{a}\right)+E_{1}\left(\frac{1}{2} e R_{a}\right)\right]
$$

where $\alpha_{0}$ is given by $(18 f)$.

As $\tilde{x}$ increases away from zero, the end effect starts to show up. Although the dominant unbounded terms in the outer limit of the inner expansions and in the inner limit of the outer expansions match each other if $\alpha$ assumes the value given in (34), no perfect functional matching can be achieved if $\mathbf{q}_{\mathbf{1}}$ is constructed from a line distribution of constant Oseenlets. On the other hand, by assuming constant Oseenlets in obtaining $q_{1}$ and matching it with $u_{0}^{*}-\mathbf{e}_{y}$ at $\tilde{x}=0$, we are stretching the range of validity and improving the accuracy of the present approximation by circumventing the end effect. As a matter of fact, the accuracy of the present approximation will be best demonstrated when we present the drag formula below. In a way we are justifying the present method a posteriori. We observe from the terms inside the curly brackets in (30) that there is a velocity component in the $x$ direction even when $\tilde{r}$ is zero. This velocity component is antisymmetric with respect to $\tilde{x}$. However, it is not present in the inner expansions $u_{0}^{*}$ as indicated by (29) when $\tilde{r}$ vanishes. That means that in the next term $\mathbf{u}_{\mathbf{1}}^{*}$ of the inner expansions we need a line distribution of Stokeslets in the 
$x$ direction, whose strengths must be antisymmetric with respect to $\tilde{x}$ as required when matching with $\mathbf{q}_{1}$. On the other hand, by a simple symmetry argument, we anticipate that there should be no net forces in the $x$ direction. Hence any distribution of Stokeslets in the $x$ direction must necessarily be antisymmetric with respect to $\tilde{x}$.

The total drag force acting on the prolate spheroid (1) is in the $y$ direction; and its magnitude can be evaluated by integrating the strengths of the distributed Oseenlets:

$$
D=4 \pi \rho \nu^{2} \int_{-e R_{a}}^{e R_{a}} 2 \alpha d \tilde{\xi}=16 \pi \mu U a e \alpha,
$$

where $\alpha$ is determined by (34). The above drag formula is quite simple analytically, and it has some interesting consequences.

In the limiting case when the Reynolds number $R_{a}$ based on the semi-major axis tends to zero, $(35)$ reduces to

$$
\begin{array}{r}
\lim _{R_{a} \rightarrow 0} D=\frac{32 \pi \mu U a e^{3}}{2 e+\left(3 e^{2}-1\right) \log [(1+e) /(1-e)]}\left[1+\frac{2 e^{3} R_{a}}{2 e+\left(3 e^{2}-1\right) \log [(1+e) /(1-e)]}\right. \\
\left.+O\left(R_{a}^{2}\right)\right] .
\end{array}
$$

Although (36) looks the same as (26b), we did not assume in deriving it that both Reynolds numbers are of the same order, $R_{b}=O\left(R_{a}\right)$, as we did in case 1 [see (17)]. In fact, (36) can also be applied when $R_{b}$ is much smaller than $R_{a}$, say

$$
R_{b}=O\left(R_{a}^{n}\right) \quad(n=2,3, \ldots) .
$$

Since the ratio $R_{b} / R_{a}$ of the two Reynolds numbers is the same as the axis ratio $b / a$ (sometimes called the slenderness parameter), $R_{b}$ being of the same order as $R_{a}$ means that the spheroid is nearly spherical. And when $R_{b}$ is much smaller than $R_{a}$, the axis ratio $b / a$ is very small, in other words the spheroid is very slender. As we noted before, the leading term in (36), that is

$$
D=\frac{32 \pi \mu U a e^{3}}{2 e+\left(3 e^{2}-1\right) \log [(1+e) /(1-e)]},
$$

is precisely the Oberbeck (1876) drag formula derived from the Stokes equations (11). If we let the axis ratio $b / a$ tend to $1,(36)$ further reduces to

$$
\lim _{R_{a \rightarrow 0, b / a \rightarrow 1}} D=6 \pi \mu U a\left(1+\frac{3}{8} R_{a}\right),
$$

which is exactly the Oseen (1910) correction to the Stokes drag formula for a sphere.

If the spheroid becomes very slender $(b / a \rightarrow 0$ or $e \rightarrow 1)$ with $R_{a}$ small, (36) yields the following result:

$$
\lim _{R_{\sigma} \rightarrow 0, b / a \rightarrow 0} D=\frac{8 \pi \mu U a}{\log (2 a / b)+\frac{1}{2}}\left[1+\frac{\frac{1}{2} R_{a}}{\log (2 a / b)+\frac{1}{2}}+O\left(R_{a}^{2}\right)\right] .
$$

Hence the drag tends to zero when the spheroid becomes very slender with $R_{a}$ also vanishing. 


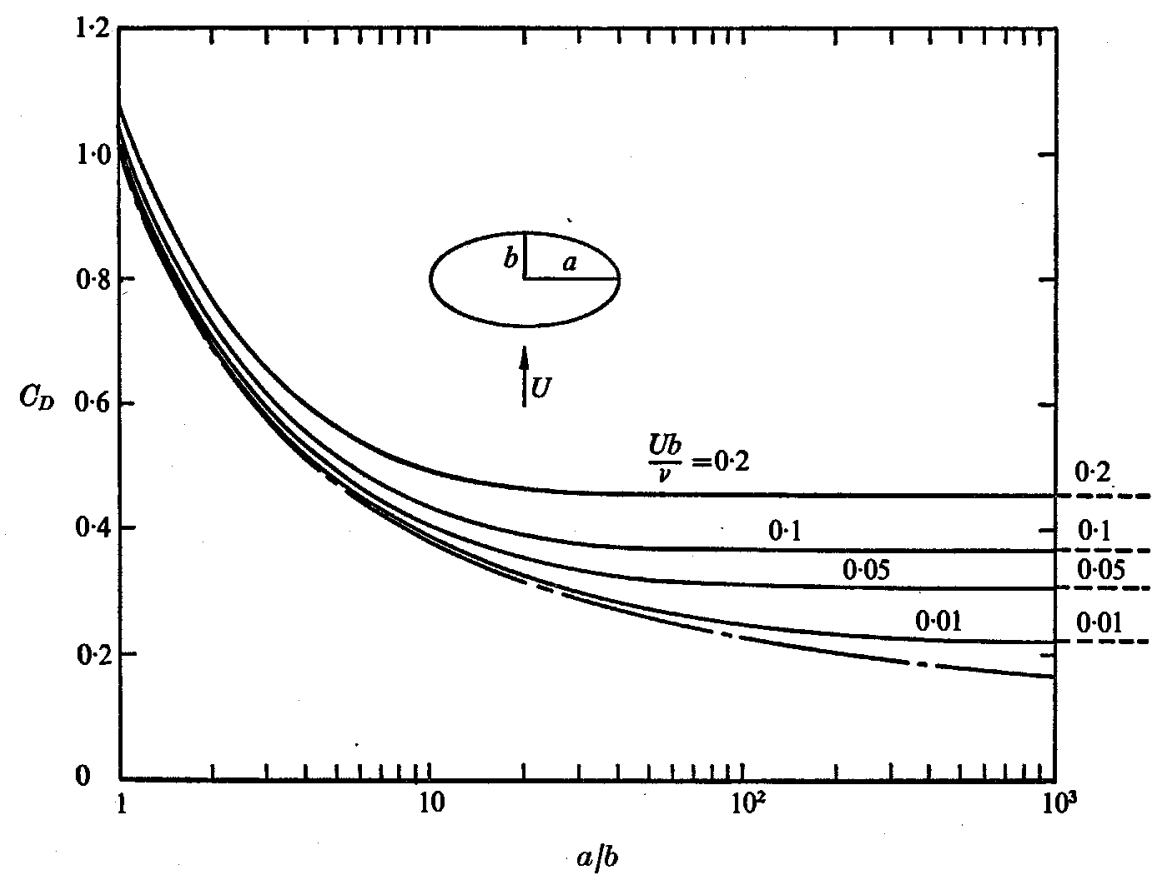

Frgure 1. The Oberbeck (or Stokes) (-- - , the Oseen (- - ) and the present (-) drag coefficient, normalized by $6 \pi \mu U a$, for uniform transverse flow of velocity $U$ past a prolate spheroid of semi-minor axis $b$ and semi-major axis $a, U b / \nu$ being the Reynolds number based on the minor axis.

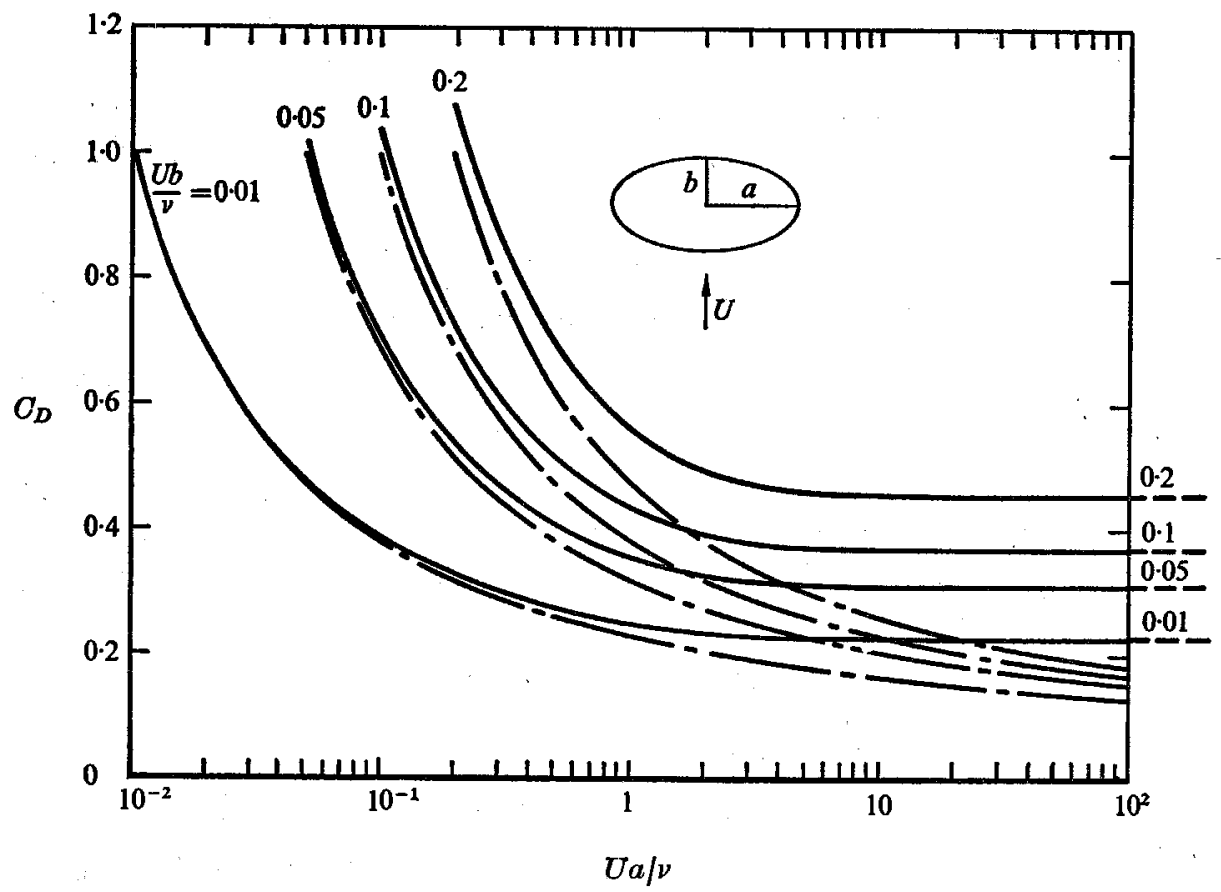

Figdre 2. Variation of the Stokes (-- - ), the Oseen (-- ) and the present $(-)$ drag coefficient with the Reynolds number $U a / \nu$ for various Reynolds numbers $U b / \nu$. 


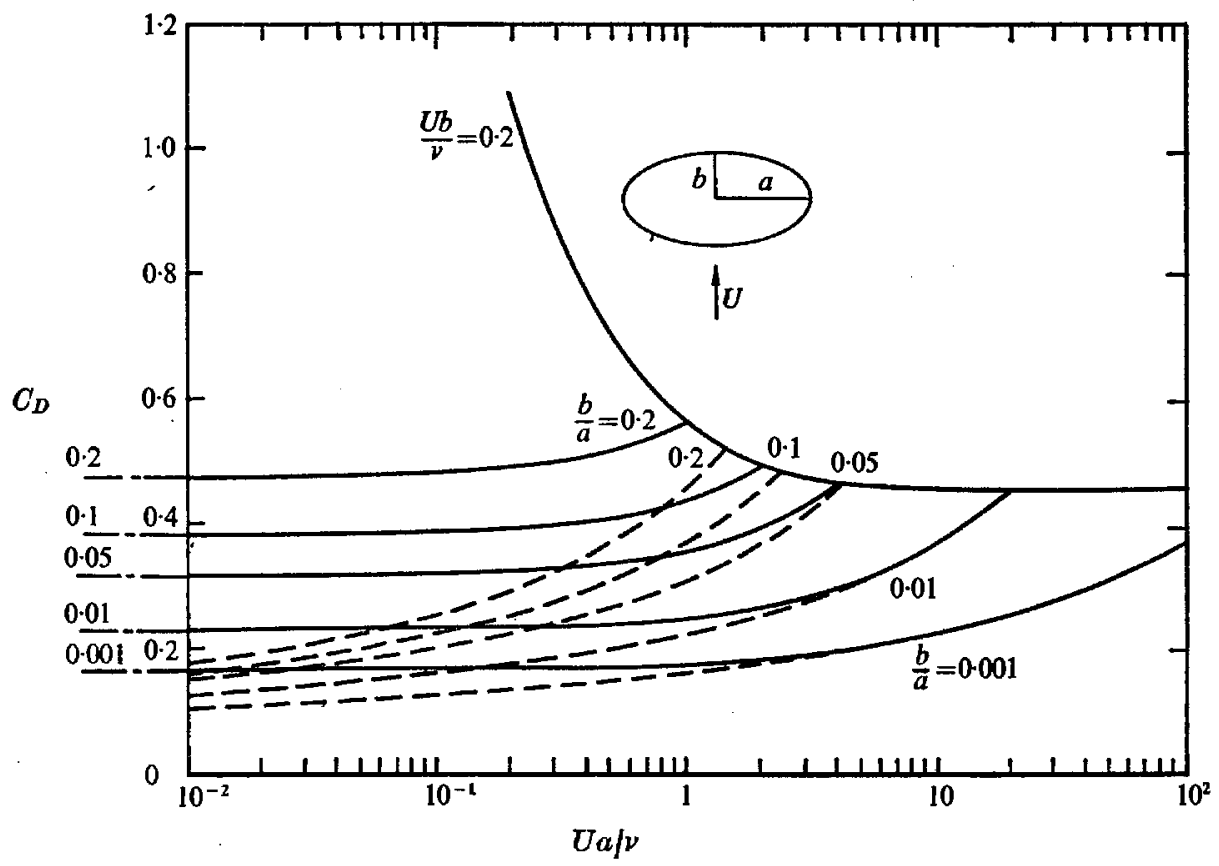

Figure 3. Variation of the Stokes (-- -), the Oseen (---) and the present $(-)$ drag coefficient with the Reynolds number $U a / v$ for various slenderness ratios $b / a$.

On the other hand, if $R_{a}$ approaches infinity, which implies that the spheroid is very slender since the slenderness parameter $b / a\left(=R_{b} / R_{a}\right)$ must necessarily be small, the drag formula (35) becomes

$$
\lim _{R_{a \rightarrow \infty}(b / a \rightarrow 0)} \frac{D}{2 a}=\frac{4 \pi \mu \dot{U}}{\log \left(4 / R_{b}\right)-\gamma+\frac{1}{2}},
$$

which agrees with the Oseen drag per unit length on an infinitely long circular cylinder of radius $b$ (Lamb 1932, p. 616). We should bear in mind that in deriving the above formulae, from (35)-(40), $R_{b}$ is always assumed small.

The present drag (35) in its coefficient form $(D / 6 \pi \mu U a)$ is plotted in figure $1 \mathrm{vs}$. the aspect ratio $a / b$ (the inverse of the slenderness ratio $b / a$ ) for several values of the Reynolds number $R_{b}$ ranging from 0.01 to 0.2 . The Oberbeck result (37) derived from the Stokos equations and the Oseen drag (40) are also plotted in their coefficient form in figure 1 for comparison. We note that the present result approaches the Oberbeck solution (designated as Stokes' solution in the figure) as $R_{b}$ decreases for fixed $a / b$. As the aspect ratio $a / b$ increases, that is when the body becomes slender, the present solution tends to that of Oseen for fixed $\boldsymbol{R}_{\boldsymbol{b}}$. However, our solution approaches to that of Stokes as $a / b$ decreases for fixed $\boldsymbol{R}_{b}$. The three drag coefficients are also plotted in figure $2 v s$. the Reynolds number $\boldsymbol{R}_{\boldsymbol{a}}$ for several values of $R_{b}$. It can be seen from figure 2 that for fixed $R_{b}$ the present result tends to Stokes' result when $R_{a}$ is very small, and to Oseen's result when $\boldsymbol{R}_{a}$ is very large. Between these two extremes our result provides a smooth transition. In figure 3 the same three drag coefficients are plotted $v s$. the Reynolds number 
$\boldsymbol{R}_{\boldsymbol{a}}$ for several slenderness ratios. It can be seen that for fixed slenderness ratio $b / a$ the present result approaches the Stokes (Oberbeck) drag if $R_{a}$ is very small; however our solution tends to Oseen's drag if $R_{a}$ is very large.

\section{Conclusions}

In the present paper we have analysed the problem of uniform transverse flow past a prolate spheroid of arbitrary aspect ratio at low values of the Reynolds number $R_{b}$ based on the semi-minor axis $b$, leaving the Reynolds number $R_{a}$ based on the semi-major axis arbitrary. A drag formula in closed analytic form has been derived by the method of matched asymptotic expansions for small $R_{b}$ and arbitrary $R_{a}$. We have neglected the end effect by assuming a line distribution of constant Oseenlets in obtaining the leading term of the outer expansions. This approximation is based on the conjecture that the end effect is not important in the leading term of the outer expansions since the body shrinks to a needle of zero radius although of finite length when viewed in the outer or Oseen variables. The end effect will probably show up in higher-order approximations. However, by assuming a line distribution of constant Oseenlets but matching it with the outer limit of the inner expansions at the mid-point of the needle, i.e. the plane $x=0$, where the end effect reduces to a minimum or even disappears because of the symmetry of the body and of the flow, we are stretching the range of validity of the present approximate solution and improving its accuracy a great deal. In fact, as the present drag formula agrees with the Oberbeck (1876) result when $R_{a}$ tends to zero and with the Oseen (1910) drag formula when $R_{a}$ tends to infinity, we have great confidence in the present solution. After a posteriori justification we may look at the present method in this way: although we do not have the correct distribution of Oseenlets, we have determined the total strength of the Oseenlets fairly accurately since the drag is derived by integrating the strengths of the distributed Oseenlets.

On the other hand, there are no general analytic methods available at present to handle problems with even one arbitrary parameter. The conventional perturbation method, either singular or regular, requires that some parameter is either very small or very large. For an arbitrary parameter, neither small nor large, perturbation techniques failed to yield anything meaningful. Therefore the present method, although not rigorously justified, provides us with a good starting point to analyse this type of problem and to produce some useful and practical solutions. To obtain an exact solution for arbitrary Reynolds number $R_{a}$ is as difficult as solving the general Navier-Stokes equations. We do not anticipate that this task can be accomplished, at least not in the near future.

Regarding the present solution for uniform transverse flow past a prolate spheroid of arbitrary aspect ratio, we note that there are two important parameters involved in this problem, namely the Reynolds number $R_{b}$ based on the semi-minor axis and the Reynolds number $R_{a}$ based on the semi-major axis. For small values of $R_{b}$, Oberbeck's drag formula gives a good approximation when $R_{a}$ is also very small; whereas the Oseen solution yields excellent results when $R_{a}$ is very large. Between these two extremes the present solution provides a 
smooth transition. In the limiting case of slender spheroids, the slenderness ratio $b / a$ alone does not determine what type of solution we are going to have, because small values of $b / a$ could mean either-that $R_{b}$ is small and $R_{a}$ is large or that both $R_{b}$ and $R_{a}$ are small with $R_{b}$ much smaller than $R_{a}$. Hence, for slender spheroids, we must know the magnitude of the Reynolds number $R_{a}$ in addition to that of $R_{b}$ in order to have an accurate estimate of the drag.

The authors are greatly indebted to Professor Sir James Lighthill for his invaluable suggestions and comments. This work was partially sponsored by the National Science Foundation and by the Office of Naval Research.

\section{REFERENCES}

Breach, D. R. 1961 Slow flow past ellipsoids of revolution. J. Fluid Mech. 10, 306-314. Chwang, A. T. 1975 Hydromechanics of low-Reynolds-number flow. Part 3. Motion of a spheroidal particle in quadratic flows. J. Fluid Mech. 72, 17-34.

ChWang, A. T. \& WU, T. Y. 1974 Hydromechanics of low-Reynolds-number flow. Part 1. Rotation of axisymmetric prolate bodies. J. Fluid Mech. 63, 607-622.

Chwang, A. T. \& WU, T. Y. 1975 Hydromechanics of low-Reynolds-number flow. Part 2. The singularity method for Stokes flows. J. Fluid Mech. 67, 787-815.

KapuON, S. \& Lagerstrom, P. A. 1957 Asymptotic expansions of Navier-Stokes solutions for small Reynolds numbers. J. Math. Mech. 6, 585-593.

LAME, H. 1932 Hydrodynamics. Cambridge University Press.

OвERвECK, A. 1876 Ueber stationäre Flüssigkeitsbewegungen mit Berücksichtigung der inneren Reibung. J. reine angew. Math. 81, 62-80.

Oswew, C. W. 1910 Über die Stokessche Formel und über die verwandte Aufgabe in der Hydrodynamik. Arkiv Math. Astron. Fys. 6, No. 29.

Proddman, I. \& Pearson, J. R. A. 1957 Expansions at small Reynolds number for the flow past a sphere and a circular cylinder. J. Fluid Mech. 2, 237-262.

S\#I, Y. Y. 1965 Low Reynolds number flow past an ellipsoid of revolution of large aspect ratio. J. Fluid Mech. 23, 657-671.

STOKEs, G. G. 1851 On the effect of the internal friction of fluids on the motion of pendulums. Trans. Camb. Phil. Soc. 9, 8-106. 